\title{
DEVELOPMENT AND EVALUATION OF NOVEL DRUG DELIVERY SYSTEM OF TOLTERODINE TARTRATE
}

\author{
VIJAYKUMAR PATIL ${ }^{*}$, DEEPAK BELSARE ${ }^{2}$
}

${ }^{1}$ Faculty of Pharmacy, Pacific Academy of Higher Education and Research University, Udaipur, ${ }^{2}$ NDMVP Samaj's College of Pharmacy, Nasik Email: patil_vijaykumar@hotmail.com

Received: 09 Mar 2017, Revised and Accepted: 22 Aug 2017

\begin{abstract}
Objective: Tolterodine tartrate (tolterodine) is used for treating overactive bladder (OAB) with symptoms of urinary frequency, urgency and leakage. Tolterodine is an antimuscarinic (anticholinergic) agent. It works by blocking a chemical that causes contractions of the bladder. Present work involved development of a novel drug delivery system of tolterodine intended to be taken once daily.
\end{abstract}

Methods: Extended release (ER) pellets of tolterodine were prepared and optimized for in vitro drug release. Subsequently, these pellets were filled into a suitable sized capsule. The resulting capsules were evaluated for in vitro drug release. Optimized formulation was subjected to accelerated stability studies for 3 mo and was evaluated for description, average weight, assay and drug release.

Results: The optimized ER capsule exhibited similar dissolution profile as that of the reference listed drug (RLD), with approximately 45\%, 75\% and more than $80 \%$ release in $3 \mathrm{~h}, 5 \mathrm{~h}$ and $7 \mathrm{~h}$ respectively. Accelerated stability studies indicated good physical and chemical stability of the formulation.

Conclusion: ER formulation of tolterodine was optimized and can be used as once a day dosage, reducing the frequency of administration when compared with the immediate release formulation. The developed formulation exhibited similar behavior as that of reference formulation Detrol LA marketed in the US.

Keywords: Tolterodine, ER, Surelease, Osmogent, Detrol LA

(C) 2017 The Authors. Published by Innovare Academic Sciences Pvt Ltd. This is an open access article under the CC BY license (http://creativecommons.org/licenses/by/4.0/) DOI: http://dx.doi.org/10.22159/ijap.2017v9i5.18381

\section{INTRODUCTION}

Tolterodine, antimuscarinic (anticholinergic) agent is indicated for treating $\mathrm{OAB}$ with symptoms of urinary frequency, urgency and leakage [1, 2]. Tolterodine acts as a competitive antagonist of acetylcholine at postganglionic muscarinic receptors. Both urinary bladder contraction and salivation are mediated via cholinergic muscarinic receptors. After oral administration, tolterodine is metabolized in the liver, resulting in the formation of 5-hydroxymethyl tolterodine (5-HMT), the major pharmacologically active metabolite. 5-HMT, which exhibits an antimuscarinic activity similar to that of tolterodine, contributes significantly to the therapeutic effect. Both tolterodine and 5-HMT exhibit a high specificity for muscarinic receptors, since both show negligible activity and affinity for other neurotransmitter receptors and other potential cellular targets, such as calcium channels [3].

The international continence society (ICS) defines incontinence as the involuntary loss of bladder or bowel control.

Urinary incontinence (UI) is a stigmatized, under-reported, underdiagnosed, under-treated condition that is erroneously thought to be a normal part of aging. One-third of men and women aged 30-70 believe that incontinence is part of aging to accept.

Information on healthy bladder function can help promote the understanding that incontinence is not a normal part of aging but a symptom of another problem.

The social costs of UI are high and even mild symptoms affect social, sexual, interpersonal, and professional function.

For urge incontinence, medications known as anticholinergics/ antimuscarinics (tolterodine, oxybutynin chloride, darifenacin, fluoxetine hydrochloride, and solifenacin succinate) can prevent bladder spasms and $\mathrm{OAB}$.

At the time of research, there was no alternative formulation available other than innovator which was patented and costly. The aim of the present study was to develop a formulation using simpler techniques and circumventing the technology used by the innovator product in order to have technological and commercial advantage along with benefit to society.

In the present research work tolterodine was selected for the development of an ER [4] formulation which was developed as a generic version of Detrol LA, marketed by Pfizer-Pharmacia and Upjohn Co.

The study was directed towards the development of a novel drug delivery system based on pelletization technique and was limited to the development of a formulation which can be commercially exploited for well-being of society in general and meant for the US market in particular.

\section{MATERIALS AND METHODS}

Chemicals and reagents

Tolterodine was procured from Cipla, Mumbai, India; surelease E-719010, sugar spheres and hypromellose 2910 USP were procured from Colorcon India Ltd, microcrystalline cellulose and mannitol were procured from Signet chemical, Mumbai, India; dibutyl sebacate was received as a gift sample from Vertellus specialties Inc., USA; hypromellose phthalate HP 55 was received as a gift sample from Shin-Etsu., Japan; isopropyl alcohol and methylene chloride was procured from Avantor, India; talc was received as a gift sample from Connell bros., Mumbai, India and empty hard gelatin capsule (EHGC) shells were received as gift samples from Associated capsules Ltd., Mumbai, India.

\section{Equipments}

rapid mixer granulator (RMG) (Gansons-3l), Gansons Ltd, Mumbai, India; extruder-sperodizer (USPH-60), Umang Pharmatech, India; fluid bed equipment (GPCG 1.1), Pam Glatt, India; blender (3l), RP products, India.

\section{Methods}

Preparation of immediate release core pellets of tolterodine

Trials to formulate the core immediate release pellets of tolterodine (table 1). 
Table 1: Composition of immediate release pellets of tolterodine

\begin{tabular}{|c|c|c|c|c|c|c|c|c|}
\hline \multirow[t]{2}{*}{ Ingredients } & \multirow[t]{2}{*}{ Function } & 4/F051A & 4/F052 & 4/F079B & 4/F113B & 4/F117D & 4/F127A & 4/F187 \\
\hline & & \multicolumn{7}{|c|}{ mg/capsule } \\
\hline \multicolumn{9}{|l|}{ Drug core by extrusion spheronisation } \\
\hline Tolterodine tartrate & Active & 4 & 4 & NA & NA & NA & 4 & 4 \\
\hline Microcrystalline cellulose (Avicel PH 101) & Diluent & 128 & 128 & NA & NA & NA & 128 & 100 \\
\hline Mannitol & Diluent & NA & NA & NA & NA & NA & NA & 28 \\
\hline Hypromellose, 2910 (Methocel E5) & Binder & 10 & 10 & NA & NA & NA & 10 & 10 \\
\hline Purified water & Solvent & qs & qs & NA & NA & NA & qs & qs \\
\hline Average weight (mg) & & 142 & 142 & NA & NA & NA & 142 & 142 \\
\hline \multicolumn{9}{|c|}{ Drug core by drug loading in fluid bed equipment } \\
\hline Tolterodine tartrate & Active & NA & NA & 3.6 & 3.6 & 3.6 & NA & NA \\
\hline Sugar spheres USNF (20-25\#) & Inert core & NA & NA & 137.25 & 137.25 & 137.25 & NA & NA \\
\hline Hypromellose, 2910 (Methocel E5) & Binder & NA & NA & 0.68 & 0.68 & 0.68 & NA & NA \\
\hline Purified water & Solvent & NA & NA & qs & qs & qs & NA & NA \\
\hline Average weight (mg) & & NA & NA & 141.53 & 141.53 & 141.53 & NA & NA \\
\hline
\end{tabular}

mg: milligram, NA: not applicable, qs: quantity sufficient

A total of 7 formulations were prepared. Extrusion and spheronisation was followed for batches 4/F051A, 4/F052, 4/F127A and 4/F187 where Tolterodine, microcrystalline cellulose and mannitol (present only in 4/F187) were sifted through 30 mesh sieve. The resultant material was mixed in a RMG for $10 \mathrm{~min}$. Methocel E5 was dissolved in water and the dry mix was granulated with this binder solution which was followed by extrusion, spheronozation and drying.
Pellets of batch numbers 4/F079B, 4/F113B and 4/F117D prepared using fluid bed coating process where Tolterodine and methocel E5 were dissolved in water. This drug solution was sprayed onto sugar spheres in fluid bed equipment and then pellets were dried.

\section{Evaluation of pellets}

The pellets of all the seven batches were evaluated for loss on drying using Mettler Toledo IR moisture analyzer.

\section{ER coating of the core pellets}

Table 2: Formulae for the ER coating of tolterodine immediate release pellets

\begin{tabular}{|c|c|c|c|c|c|c|c|c|}
\hline \multirow[t]{2}{*}{ Ingredients } & \multirow[t]{2}{*}{ Function } & 4/F051A & 4/F052 & 4/F079B & 4/F113B & 4/F117D & 4/F127A & 4/F187 \\
\hline & & \multicolumn{7}{|c|}{ mg/capsule } \\
\hline \multicolumn{9}{|l|}{ ER coating } \\
\hline $\begin{array}{l}\text { Tolterodine tartrate drug coated } \\
\text { pellets }\end{array}$ & Drug coated pellets & 142 & 142 & 141.53 & 141.53 & 141.53 & 142 & 142 \\
\hline Surelease E-7-19010 & Rate controlling polymer & 21.25 & 21.25 & 28.8 & 19.8 & 18 & 21.875 & 35 \\
\hline Hypromellose, 2910 (Methocel E5) & Channeling agent & 3.75 & 3.75 & 3.2 & 2.2 & 2 & 3.123 & NA \\
\hline Purified water & Solvent & qs & qs & qs & qs & qs & qs & qs \\
\hline Average weight (mg) & & 167 & 167 & 173.53 & 163.53 & 161.53 & 167 & 177 \\
\hline
\end{tabular}

mg: milligram, ER: Extended release, qs: quantity sufficient

Methocel was dissolved in water which was later added into surelease dispersion, this resultant dispersion was sprayed onto

immediate release drug pellets into fluid bed equipment followed by drying.

Delayed release (DR) and immediate release (IR) coating of the ER pellets

Table 3: Formulae for the DR coating of tolterodine ER pellets

\begin{tabular}{|c|c|c|c|c|c|c|c|c|}
\hline \multirow[t]{2}{*}{ Ingredients } & \multirow[t]{2}{*}{ function } & 4/F051A & 4/F052 & 4/F079B & 4/F113B & 4/F117D & 4/F127A & 4/F187 \\
\hline & & \multicolumn{7}{|c|}{ mg/capsule } \\
\hline \multicolumn{9}{|l|}{ DR coating } \\
\hline Tolterodine ER coated pellets & ER coated pellets & NA & 167 & NA & NA & NA & 167 & NA \\
\hline Hypromellose phthalate HP55 & Enteric polymer & NA & 6 & NA & NA & NA & 6 & NA \\
\hline Dibutylsebacate, NF & Plasticizer & NA & 0.8 & NA & NA & NA & 0.8 & NA \\
\hline Hypromellose, 2910 USP (Methocel E5) & Channeling agent & NA & 2 & NA & NA & NA & 2 & NA \\
\hline Talc, USP & Anti-tacking agent & NA & 1.2 & NA & NA & NA & 1.2 & NA \\
\hline Isopropyl alcohol, USP & Solvent & NA & qs & NA & NA & NA & qs & NA \\
\hline Methylene chloride, NF & Solvent & NA & qs & NA & NA & NA & qs & NA \\
\hline Average weight (mg) & & - & 177 & - & - & - & 177 & - \\
\hline \multicolumn{9}{|l|}{ IR coating } \\
\hline Tolterodine ER coated pellets & ER coated pellets & NA & NA & 173.53 & 163.53 & 161.53 & NA & NA \\
\hline Tolterodine tartrate & Active & NA & NA & 0.4 & 0.4 & 0.4 & NA & NA \\
\hline Hypromellose, 2910 (Methocel E5) & Binder & NA & NA & 0.07 & 0.07 & 0.07 & NA & NA \\
\hline Purified water & Solvent & NA & NA & qs & qs & qs & NA & NA \\
\hline Average weight (mg) & & - & - & 174 & 164 & 162 & - & - \\
\hline \multicolumn{9}{|l|}{ Blending } \\
\hline Purified talc NF & Glidant & 2 & 2 & 2 & 2 & 2 & 2 & 2 \\
\hline Average weight (mg) & & 169 & 179 & 176 & 166 & 164 & 179 & 179 \\
\hline \multicolumn{9}{|l|}{ Capsule filling } \\
\hline EHGC 1 No. & & 43 & 43 & 43 & 43 & 43 & 43 & 43 \\
\hline Total weight of capsule (mg) & & 212 & 222 & 219 & 209 & 207 & 222 & 222 \\
\hline
\end{tabular}

DR: delayed release, NA: not applicable, qs: quantity sufficient, mg: milligram, IR: immediate release 
Hypromellose phthalate and Methocel E5 were dissolved in a mixture of isopropyl alcohol and methylene chloride. Dibutyl sebacate was added to this solution followed by dispersion of talc. This dispersion was sprayed on Tolterodine ER pellets of batch number 4/F052 and 4/F127A in fluidized bed equipment and then dried. Dried pellets were blended with talc and filled into capsules shells

\section{Reproducibility trial and accelerated stability studies}

Batch 4/F191 was prepared similar to the composition of the batch 4/F187 in order to evaluate reproducibility and the stability profile of the formulation. The capsules were packed in 30cc HDPE bottles and subjected to accelerated storage conditions of $40{ }^{\circ} \mathrm{C} / 75 \%$ relative humidity. Samples were evaluated at the time intervals of 1 , 2 and 3 mo.

\section{Statistical analysis}

One way analysis of variance (ANOVA) was employed to assess the difference between the assay values of initial and that of stability samples using Sigma Stat software (Sigma stat 2.03, SPSS). The observed $p$ values of $>0.05$ were considered statistically significant for the test. Similar statistical test was applied to find difference in the in vitro drug release at each time point among stability samples.

\section{RESULTS AND DISCUSSION}

The aim of the present study was to develop an ER formulation of tolterodine which can be used as once a day therapy and was developed as a generic version of Detrol LA marketed by PfizerPharmacia and Upjohn Co. by employing pelletization technique and layer building method.
Several trials were conducted (as shown in table 1,2 and 3) using different \% of ER coating, employing DR coating on to the ER coating and changing the ratio of ER polymer to channel former.

As seen from the data, drug release from all the trials having batch numbers 4/F051A, 4/F052, 4/F079B, 4/F113B, 4/F117D and 4/F127A with ER polymer ranging between $18 \mathrm{mg}$ to $21.875 \mathrm{mg}$ were slow in release compared to Detrol LA. Further, the batches 4/F052 and 4/F127A with DR coating over ER coating have not shown much improvement with respect to drug release. The batches 4/F079B, 4/F113B, 4/F117D with IR coating on ER coating have not shown much improvement with respect to drug release. Surprisingly batch number 4/F187 with highest ER polymer of $35 \mathrm{mg}$ showed more drug release compared to other batches and was similar to the drug release profile of Detrol LA.

The ER coating of batch 4/F187 was formulated without a channel former. Surprisingly it showed improvement in release. This was attributed to the presence of mannitol in core pellets of the batch 4/F187, which has helped in dissolution of drug in the core and this solution in the core has created an osmotic effect. Since there was no channel former in the outer ER layer, the ethyl cellulose present in the outer layer was forced to swell and the osmotic pressure from core pellets expelled the drug in a controlled fashion through the swollen ethyl cellulose layer. There are references which mentions that osmotic pressure created by using osmotic agent/osmogent $[5$, 6] causes pellets to burst and dump the drug [7], however the tolterodine formulation developed as pellets in this study does not cause the burst and does not dump the drug, this is evident by similar dissolution of the formulation 4/F187 (with an osmotic agent) compared against reference product Detrol LA where osmotic agent is not part of formulation.

Table 4: Evaluation of capsule formulations of tolterodine

\begin{tabular}{|c|c|c|c|c|c|c|c|c|}
\hline Evaluation parameter & $\begin{array}{l}\text { C100378 } \\
\text { Detrol LA }\end{array}$ & 4/F051A & 4/F052 & 4/F079B & 4/F113B & 4/F117D & 4/F127A & 4/F187 \\
\hline Average weight (mg) & $229 \pm 2.2$ & $212 \pm 2.7$ & $222 \pm 1.7$ & $218.53 \pm 2.1$ & $208.53 \pm 2.5$ & $206.53 \pm 1.9$ & $222 \pm 0.9$ & $222 \pm 1.8$ \\
\hline Lock length (mm) & $15.9 \pm 0.3$ & $15.9 \pm 0.3$ & $15.9 \pm 0.3$ & $15.9 \pm 0.3$ & $15.9 \pm 0.3$ & $15.9 \pm 0.3$ & $15.9 \pm 0.3$ & $15.9 \pm 0.3$ \\
\hline \multirow{2}{*}{ Assay (\%) } & $100.2 \pm 0.9$ & 99.5 & 99.8 & 100.3 & 99.5 & 100.8 & 100.3 & 99.5 \\
\hline & & \pm 2.2 & \pm 2.2 & \pm 2.7 & \pm 1.8 & \pm 0.9 & \pm 2.7 & \pm 2.2 \\
\hline Drug release at $1 \mathrm{~h}(\%)$ & $8 \pm 2.1$ & $8 \pm 2.2$ & $7 \pm 0.9$ & $10 \pm 0.9$ & $12 \pm 0.9$ & $13 \pm 4.5$ & $7 \pm 2.2$ & $8 \pm 1.8$ \\
\hline Drug release at $3 \mathrm{~h}(\%)$ & $45 \pm 0.8$ & $40 \pm 1.8$ & $40 \pm 1.8$ & $20 \pm 01.2$ & $24 \pm 0.9$ & $29 \pm 2.2$ & $40 \pm 1.8$ & $43 \pm 0.9$ \\
\hline Drug release at $5 \mathrm{~h}(\%)$ & $73 \pm 2.3$ & $58 \pm 0.9$ & $64 \pm 0.9$ & $33 \pm 2.2$ & $38 \pm 2.8$ & $44 \pm 3.1$ & $66 \pm 0.9$ & $74 \pm 2.7$ \\
\hline Drug release at $7 \mathrm{~h}(\%)$ & $81 \pm 1.2$ & $66 \pm 4.5$ & $75 \pm 3.6$ & $44 \pm 2.1$ & $50 \pm 1.9$ & $56 \pm 1.8$ & $77 \pm 1.2$ & $83 \pm 1.8$ \\
\hline Drug release at $9 \mathrm{~h}(\%)$ & $85 \pm 1.9$ & $70 \pm 1.8$ & $82 \pm 4.5$ & $52 \pm 0.9$ & $60 \pm 2.2$ & $65 \pm 1.2$ & $83 \pm 0.9$ & $85 \pm 3.1$ \\
\hline Drug release at $12 \mathrm{~h}(\%)$ & $86 \pm 0.9$ & $78 \pm 3.9$ & $89 \pm 0.9$ & $62 \pm 2.2$ & $69 \pm 0.9$ & $74 \pm 0.9$ & $91 \pm 1.8$ & $86 \pm 1.4$ \\
\hline
\end{tabular}

mm: millimeter, h: h, mg: milligram, Values of assay and drug release are represented as mean \pm standard deviation, $\mathrm{n}=3$, $\mathrm{n}=6 \mathrm{respectively}$

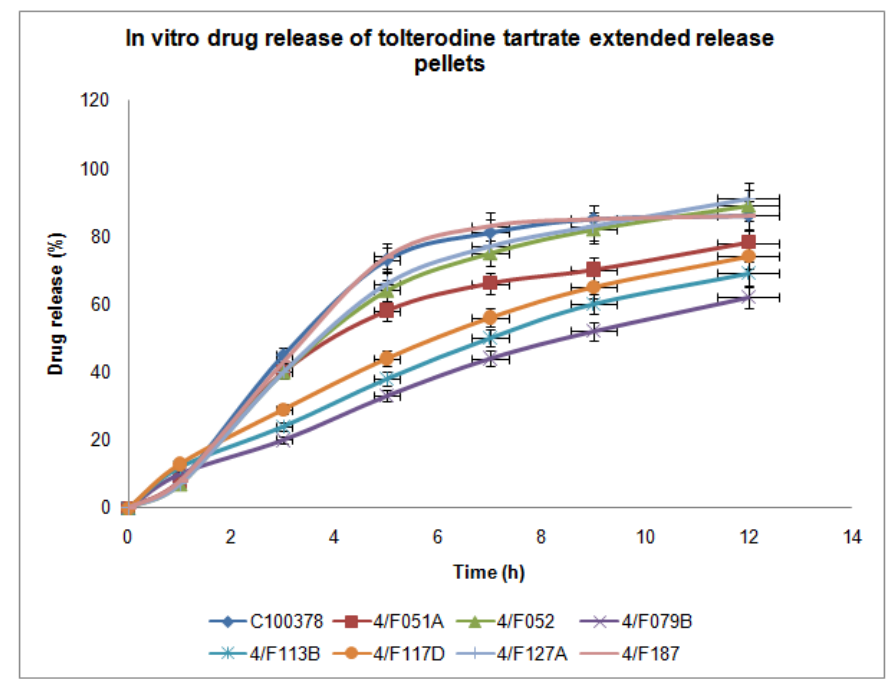

Fig. 1: In vitro drug release of tolterodine tartrate from Extended Release pellets prepared with different palletization techniques and different composition, (Values of in vitro drug release are represented as mean, $n=6$ ). 


\section{Accelerated stability studies}

Batch 4/F191 a reproducibility batch of 4/F187 behaved similar as that of batch 4/F187. Since batch 4/F187 was having similar dissolution profile as that of RLD (C100378) and batch 4/F191 was reproducibility batch of $4 / F 187$, it was chosen for stability studies. Stability samples of batch 4/F191 when evaluated at various time intervals, showed no significant difference in appearance or other physical traits as compared to initial samples (table 6). Statistical analysis of assay values of stability samples indicated no significant difference. In vitro release profiles of samples when compared with that of initial using ANOVA exhibited no significant difference thus indicating overall good stability of the formulation at accelerated conditions.

Table 6: Evaluation of stability samples of batch 4/F191

\begin{tabular}{|c|c|c|c|c|c|}
\hline Test & Specification & Initial & $1 \mathrm{mo}$ & $2 \mathrm{mo}$ & $3 \mathrm{mo}$ \\
\hline Description & White to off-white colored capsules. & complies & complies & complies & complies \\
\hline Average weight & $222 \mathrm{mg} \pm 5 \%$ & complies & complies & complies & complies \\
\hline Assay & $90-110 \%$ & $99.8 \pm 0.7$ & $100.3 \pm 1.7$ & $99.3 \pm 1.1$ & $99.1 \pm 2.0$ \\
\hline \multirow[t]{3}{*}{ Drug release } & $3 \mathrm{~h}: 45 \pm 10 \%$ & $43 \pm 0.9$ & $43.9 \pm 2.7$ & $46.4 \pm 3.5$ & $42.6 \pm 3.2$ \\
\hline & 5 h: NLT 70\% & $74 \pm 2.7$ & $73.9 \pm 2.7$ & $76.4 \pm 3.5$ & $72.6 \pm 3.2$ \\
\hline & $7 \mathrm{~h}: \mathrm{NLT} 80 \%$ & $83 \pm 1.8$ & $93.2 \pm 1.4$ & $95.2 \pm 2.5$ & $91.5 \pm 1.9$ \\
\hline
\end{tabular}

Values of assay and drug release are represented as mean \pm standard deviation, $n=3, n=6$ respectively

\section{CONCLUSION}

ER pellets prepared using mannitol as an osmotic agent/osmogent have shown the in vitro release of the drug similar to Detrol LA and subsequently exhibited complete release (more than $85 \%$ ) at $12 \mathrm{~h}$. This osmotic ER controlled formulation of tolterodine when administered once a day can thus be expected to achieve similar therapeutic effect as that of Detrol LA.

\section{ACKNOWLEDMENT}

Authors would like to acknowledge Vertellus specialties inc, USA Shin-Etsu, Japan; Connell bros., Mumbai, India and Associated capsules Ltd., Mumbai, India for generously providing the gift samples of dibutyl sebacate, hypromellose phthalate HP 55, talc and EHGC shells respectively.

\section{CONFLICT OF INTERESTS}

Declare none

\section{REFERENCES}

1. Patient information leaflet Detrol $\circledR^{\circledR}$ LA capsules submitted to US FDA-reference ID-3168106; 2005. p. 1-2.

2. Center for drug evaluation and research, medical review: application no. 21-228 S006. Applicant: Pharmacia and Upjohn Company; 2013. p. 1-3.
3. Product monograph Detrol ${ }^{\circledR}$ LA, Pfizer Canada Inc., Submission Control No: 180260; 2015. p. 1-32.

4. Umesh Nandkumar Khatavkar, K Jayaram Kumar, Shamkant Laxman Shimpi. Novel approaches for the development of oral controlled release compositions of galantamine hydrobromide and paroxetine hydrochloride hemihydrate: a review. Int J Appl Pharm 2016;8:1-6.

5. Panchaxari Mallappa Dandagi, Chirag Prakashbhai Patel, Rohit Sharma, Anand Panchakshari Gadad, Vinayak Mastiholimath. Studies on formulation and evaluation of osmotically controlled drug delivery system of carbamazepine. Int J Pharm Pharm Sci 2014;6:239-50.

6. Dehghan MHG, Kazi MS, Ansari MA. Formulation and evaluation of once daily osmotic tablet of Ketoprofen. Int J Pharm Pharm Sci 2014;6:951-7.

7. Borra SP. Formulation of tolterodine tartrate controlled release MUPS tablets by using novel core and studying the effect of protective plasticizer. Am J PharmTech Res 2016;6:632-50.

\section{How to cite this article}

- Vijaykumar Patil, Deepak Belsare. Development and evaluation of novel drug delivery system of tolterodine tartrate. Int J Appl Pharm 2017;9(5):29-32. 